\title{
MOBILITY OF NONSTEROIDAL ANTI-INFLAMMATORY DRUGS IN SOILS WITH AND WITHOUT AMENDMENT OF BIOSOLID
}

\author{
LORETO ASCAR**, INÉS AHUMADA ${ }^{a}$, NATALIA MORALES ${ }^{a}$, \\ TATIANA GARRIDO ${ }^{a}$, ADY GIORDANO ${ }^{b}$ Y KARLA LEIVA ${ }^{a}$ \\ a Departamento de Química Inorgánica y Analitica, Facultad de Ciencias Químicas y Farmacéuticas. Universidad de Chile. \\ Sergio Livingstone 1007, Independencia, Casilla 233, Santiago, Chile. \\ ${ }^{b}$ Departamento de Química Inorgánica, Facultad de Química, Pontificia Universidad Católica de Chile. \\ Av. Vicuña Mackenna 4860, Casilla 306, Correo 22, Macul, Santiago, Chile
}

\begin{abstract}
Nonsteroidal anti-inflammatory drugs are widely used by humans as therapeutic agents to treat or prevent illnesses. Once ingested, part of them is excreted and enters the wastewater treatment plants generating biosolids that they may be used as fertilizing complements.

The purpose of this study was to investigate the mobility of nonsteroidal anti-inflammatory drugs (ketoprofen, naproxen, diclofenac and ibuprofen) in soils and soils amended with biosolid.

Studies of NSAIDs adsorption on two different soils were carried through batch equilibrium at room temperature. The mobility of NSAIDs in soils was studied through the use of leaching columns incorporating in the top section soil enriched with NSAIDs with and without biosolid.

Ketoprofen, naproxen and ibuprofen were determined in the leaching columns. Anti-inflammatory mobility in the soil profile depends on the specific characteristics of each system soil-biosolid. In both soils there was little mobility of anti-inflammatory in the soil profile. In Linderos soil were found in greater quantity of Ibuprofen, naproxen and diclofenac that in Lonquén soil.
\end{abstract}

Keywords: Anti-inflammatory drugs, mobility, solid-phase extraction, HPLC-DAD.

\section{INTRODUCTION}

Nonsteroidal anti-inflammatory drugs (NSAIDs) are among highly consumed drugs by human beings all over the world ${ }^{1-2}$. They are commonly used, readily available compounds, in many cases sold over the counter. ${ }^{3}$ They are considered emerging contaminants which are present at low concentrations, but their increasing use for human and animal ${ }^{4-5}$ due to their pharmacological characteristics, painkiller, anti-inflammatory and antipyretic action; can become harmful. The most commonly utilized NSAIDs include ketoprofen, naproxen, diclofenac, and ibuprofen, with different chemical structures but similar properties and characteristics. ${ }^{6}$

The human body is able to metabolize an important percentage of the ingested NSAIDs, while the remaining percentage, together with the metabolites resulting from digestion, is excreted reaching wastewater treatment plants. ${ }^{7}$ Recent studies have clearly demonstrated that wastewater treatment plants achieve partial elimination of drugs, with percentages ranging from 20 to $90 \%$. As a result, effluents may be one of the main sources of emission of these compounds and their metabolites to the environment. ${ }^{8-12}$ On the other hand, at the end of the treatment, a fraction of these compounds may be retained in the biosolids, solid or semisolid residues rich in organic matter, microorganisms, minerals, and chemical compounds. These subproducts, high organic matter content, may contribute to improve the physical conditions of soils. However, incorporation of biosolids to soil may cause that possible present contaminants, as pharmaceutical compounds, can be degraded or absorbed in the surface soil layers, or leached to ground water and thus, they may affect the environment as a result of the phenomenon of bioaccumulation and toxicity of the drug in these soils.

The process of adsorption of a compound on soil is related to a series of phenomena that may occur along the soil profile. NSAIDs entering soil may be adsorbed, which directly affects the possibilities of chemical or biological degradation of the compound, the latter mainly as a result of the action of present microorganisms. On the other hand, potential leaching depends on the equilibrium existing in the soil-solution interfacel; if such potential is high; the possibility of groundwater contamination will also increase, which may be assessed through studies of mobility in soils.

The mobility of a compound in soil is mainly associated to leaching feasibility, which in turn depends on factors related to the physical properties such as $\mathrm{pH}$, organic matter content, humidity, texture, presence of active microbial flora that permits product biotransformation or retention in soil. Thus, biosolid incorporation to agricultural soils might affect the mobility of NSAIDs contained in the biosolid along the soil profile, being eventually able to contami- nate the phreatic level.

The present study assesses the mobility of NSAIDs in soils, by utilizing leaching columns in soils with or without incorporation of biosolid.

\section{EXPERIMENTAL}

\section{Reagents}

Ketoprofen, naproxen, diclofenac, and ibuprofen (97-100\% purity) were obtained from Sigma-Aldrich. Monthly preparation was carried out of $500 \mathrm{mg}$ $\mathrm{L}^{-1}$ solutions of each of the analytes in methanol, stored at $4{ }^{\circ} \mathrm{C}$ in the dark to prevent possible decomposition.

The solvents employed (methanol, acetone, acetonitrile, n-hexane, and ethyl acetate) were obtained from Merck, HPLC grade. Potassium dihydrogen phosphate was Merck, analytical grade. Nitrogen gas was extrapure grade $(99.995 \%)$. All the solutions were prepared with Milli-Q water (Millipore Corporation, USA) with $18 \mathrm{M} \Omega \mathrm{cm}$ conductivity. Waters.

$60 \mathrm{mg}$ Oasis HLB were used for solid phase extraction and purchased at

\section{Instrumentation}

NSAIDs determination was performed by liquid chromatography with diode array detector (HPLC-DAD) on a Waters HPLC instrument provided with binary pump (waters 1525) Atlantis column dC18 (5 $\mu \mathrm{m} ; 250 \mathrm{~mm}$ x 4.6 $\mathrm{mm}$ ), diode array detector (Waters 2998) and a Rheodyne manual injector valve, model $7725 \mathrm{i}$ with $20 \mathrm{uL}$ sample loop. Chromatograms were processed with Empower software. Mobile phase was a mixture $50 \% \mathrm{v} / \mathrm{v}$ acetonitrile: potassium dihydrogen phosphate $50 \mathrm{mM}, \mathrm{pH} 4.2$ at a flow rate of $1 \mathrm{~mL} \mathrm{~min}^{-}$ 1. NSAIDs were measured at a wavelength of $220 \mathrm{~nm}$. Calibration curves between 0.5 and $20 \mathrm{mg} \mathrm{L}^{-1}$ were performed.

\section{Samples of soils and biosolids,}

The soils samples were obtained from the surface layer $(0-20 \mathrm{~cm})$ of agricultural soils in the Metropolitan Region, corresponding to the Linderos (loamy texture) and Lonquén (clay sandy loamy texture) series. Biosolid was from a wastewater treatment plant in the Metropolitan Region which follows a process that permits concentration and stabilization before final disposal. Both soil and biosolid samples were stored in plastic bags for transportation to the laboratory, then were air-dried, disaggregated, sieved $(2 \mathrm{~mm})$ and stored in high density polyethylene jars at room temperature.

In order to decrease microbial activity of the systems, and to assess its effect on NSAIDs mobility, a portion of each soils was sterilized by high- 
pressure autoclave at a constant temperature $\left(121^{\circ} \mathrm{C}, 103 \mathrm{kPa}\right)$, for $45 \mathrm{~min}$ the first day and $30 \mathrm{~min}$ the second day. ${ }^{13}$

Characterization of soil and biosolid samples.

$\mathrm{pH}$ was determined in the supernatant of a soil-water suspension 1:2.5 $(\mathrm{w} / \mathrm{v})$ ratio. Organic matter content was determined by the Metson method using spectrophotometry at $600 \mathrm{~nm}$. Cation exchange capacity (CEC) was determined by the sodium acetate method at $\mathrm{pH}$. Soil texture was determined by the hydrometer method. ${ }^{14}$

\section{Adsorption of NSAIDs on soil}

Studies of NSAID adsorption on soil were carried out in order to select the concentration of NSAIDs to carry out mobility tests on soil samples. These studies were done through batch equilibrium at room temperature. Five $g$ of soil with different NSAIDs rates (from 0 to $500 \mathrm{mg} \mathrm{L}^{-1}$ ) were equilibrated in $25 \mathrm{~mL}$ of $\mathrm{CaCl}_{2} 0.01 \mathrm{~mol} \mathrm{~L}^{-1}$ solution (to keep ionic strength of the medium constant) and $5 \mathrm{~g}$ of $\mathrm{NaN}_{3}$ (to suppress microbial activity during equilibrium). Tubes were shaken in a reciprocal mechanical agitator for $24 \mathrm{~h}$. After centrifugation, the aqueous portion was filtered and clean-up with OASIS HLB cartridge. ${ }^{15}$ Quantification was performed by HPLC - DAD.

\section{Mobility of NSAIDs in leaching columns}

In order to achieve a higher content of NSAIDs in soil samples with similar physical and chemical characteristics, solutions of each anti-inflammatory were incorporated into the soil.

Studies of NSAIDs mobility in soil were performed on acrylic leaching columns, $12 \mathrm{~cm}$ long and $2.5 \mathrm{~cm}$ inner diameter (ID). Each column was divided into six equal $2 \mathrm{~cm}$ sections. The top section of the column contained $6 \mathrm{~g}$ of soil or sterilized soil, biosolid (0 or $\left.200 \mathrm{Mg} \mathrm{ha}^{-1}\right)$ and the equivalent to 200 mg of each NSAIDs per kg of soil (previously, they were kept in incubator to field capacity for 7 days at room temperature). Soil or sterilized soil was incorporated to the other next four sections, and quartz to the bottom section. The columns were filled and humidified, kept at room temperature for 15 days, covered with aluminum foil. They were daily humidified with $3 \mathrm{~mL} \mathrm{CaCl} 20.5$ mol L-1 and the columns were covered at the top to reduce any losses due to evaporation. ${ }^{16}$ At the end of this period, each column section was dried for further extraction of the NSAIDs from soil.

NSAIDs extraction methodology from soils and biosolids

NSAIDs extraction from soils was carried out with ultrasound assisted solvent extraction and further clean-up by means of solid-phase extraction using OASIS HLB cartridges.

$4 \mathrm{~g}$ soil from each section was transferred to glass centrifuge tubes and consecutively extracted with $20 \mathrm{~mL}$ methanol, $10 \mathrm{~mL}$ methanol, and twice with $10 \mathrm{~mL}$ acetone for 5 -min periods, with centrifugation between each stage. The supernatants of each extraction stage were collected together and filtered. Finally, the solvents were reduced in a rotary evaporator at $40{ }^{\circ} \mathrm{C}$ and were reconstituted in $100 \mathrm{~mL}$ of water. ${ }^{17}$

Clean-up with OASIS HLB cartridge for eliminate interfering compounds of different polarity to analytes that might interfere with the determination by chromatography. The cartridges were mounted on a vacuum manifold provided with manometer control. Three $1 \mathrm{~mL}$ ethyl acetate portions were passed through the cartridge, followed by three $1 \mathrm{~mL}$ portions methanol and three $1 \mathrm{~mL}$ portions Milli Q water. Next, the reconstituted aqueous sample was passed at $1 \mathrm{~mL} \mathrm{~min}^{-1}$ and then the cartridge was washed with 3 portions of 1 $\mathrm{mL}$ methanol:water $(5: 95 \% \mathrm{v} / \mathrm{v})$ and 3 portions $1 \mathrm{~mL}$-hexane. Finally, elution of NSAIDs from the cartridges was carried out with 3 aliquots of $1 \mathrm{~mL}$ ethyl acetate transferred into an amber glass vial, taken to dryness with nitrogen gas, and finally reconstituted with $0.5 \mathrm{~mL}$ methanol for further chromatographic quantification. $^{18}$

\section{RESULTS AND DISCUSSION}

Characterization of soils and biosolid:

Both soils have clay sandy loamy texture, presenting the Linderos soil a slightly higher sand content than Lonquen. Table 1 shows the results of chemical characterization of soils and biosolid. Biosolid is slightly more acidic and has higher organic matter content than both soils samples; which might affect the NSAIDs retention processes during leaching.

According to Chilean regulatory standards, soils and biosolid are below maximum allowed metal concentrations (results not shown), which would allow the application of the biosolid to soils under study.

Both soils have clay sandy loamy texture, presenting the Linderos soil a slightly higher sand content than lonquén.

Table 1. Characterization of Lonquén and Linderos soils and biosolid.

\begin{tabular}{|l|c|c|c|}
\hline & Biosolid & Lonquén & Linderos \\
\hline pH & 6.95 & 7.56 & 7.73 \\
\hline OM (\%) & 15.16 & 2.85 & 2.72 \\
\hline CEC (cmol kg-1) & 89.13 & 31.15 & 18.45 \\
\hline Clay (\%) & - & 21.5 & 21.6 \\
\hline Loam (\%) & - & 23.6 & 19.2 \\
\hline Sand (\%) & - & 54.9 & 59.2 \\
\hline
\end{tabular}

Extraction of NSAIDs from soil

In order to assess the adsorption and mobility of NSAIDs in soil soil-biosolid samples, an analytical quantification method was implemented. Ultrasoundassisted solvent extraction in soil samples enriched at concentrations of 1.2 and $2.0 \mathrm{mg} \mathrm{L}^{-1} \mathrm{NSAIDs}$ resulted in recovery percentages ranging from $68 \%$ to $99 \%$, indicating that the extraction method is adequate to determine NSAIDs in soil samples (Table 2). The highest recoveries were to diclofenac and ibuprofen, which correspond to the more apolar studied compounds.

Table 2. Recovery of the method ultrasound-assisted extraction in soil enriched with NSAIDs at enriched 1.2 and $2.0 \mathrm{mg} \mathrm{L}^{-1}$.

\begin{tabular}{|c|c|c|}
\hline & \multicolumn{2}{|c|}{ Recovery (\%) } \\
\hline & $\mathbf{1 . 2} \mathbf{~ m g ~ L ~}^{-\mathbf{1}}$ & $\mathbf{2 . 0} \mathbf{~ m g ~ L}^{-\mathbf{1}}$ \\
\hline Ketoprofen & 84 & $\mathbf{7 4}$ \\
\hline Naproxen & 68 & 74 \\
\hline Diclofenac & 92 & 88 \\
\hline Ibuprofen & 93 & 99 \\
\hline
\end{tabular}

A chromatogram of an extract of Linderos soil enriched with NSAIDs is presented in Figure 1. A linear behavior was observed for the four NSAIDs up to concentrations of $50 \mathrm{mg} \mathrm{L}^{-1}$, with linear correlation coefficients above 0.9996 for all of the analytes under study. Detection limits of method ranged from 5.8 to $35.4 \mathrm{mg} \mathrm{L}^{-1}$ and limits of quantification were between 19.2 and $118.2 \mathrm{mg} \mathrm{L}^{-1}$ for the different analytes. ${ }^{19}$ Presence of anti-inflammatory in soils in study and biosolid was determined, only was found naproxen in biosolid at a concentration of $0.023 \pm 0.001 \mu \mathrm{g} \mathrm{g}^{-1}$.

\section{Adsorption capacity of NSAIDs in soi}

Adsorption studies were interpreted through studies of adsorption isotherms ${ }^{20}$ and a type $\mathrm{S}$ isotherm behavior was observed. Adjustment was performed by use to the Freundlich and Langmuir models, and it was found that studies only adjusted to the Freundlich model, with correlation coefficien values for the different NSAIDs ranging from 0.78 to 0.93 (Table 3 ). From these results, it may be stated that adsorption isotherms of the NSAIDs under study can be best described with the Freundlich equation. Xu et al., found that adsorption isotherms of NSAIDs in 4 types of soil also adjusted to the Freundlich equation. ${ }^{21} \mathrm{Kd}$ values obtained (Table 3 ) indicate that ibuprofen was the least adsorbed, while adsorption of diclofenac, ketoprofen and naproxen on soil shows similar values. 


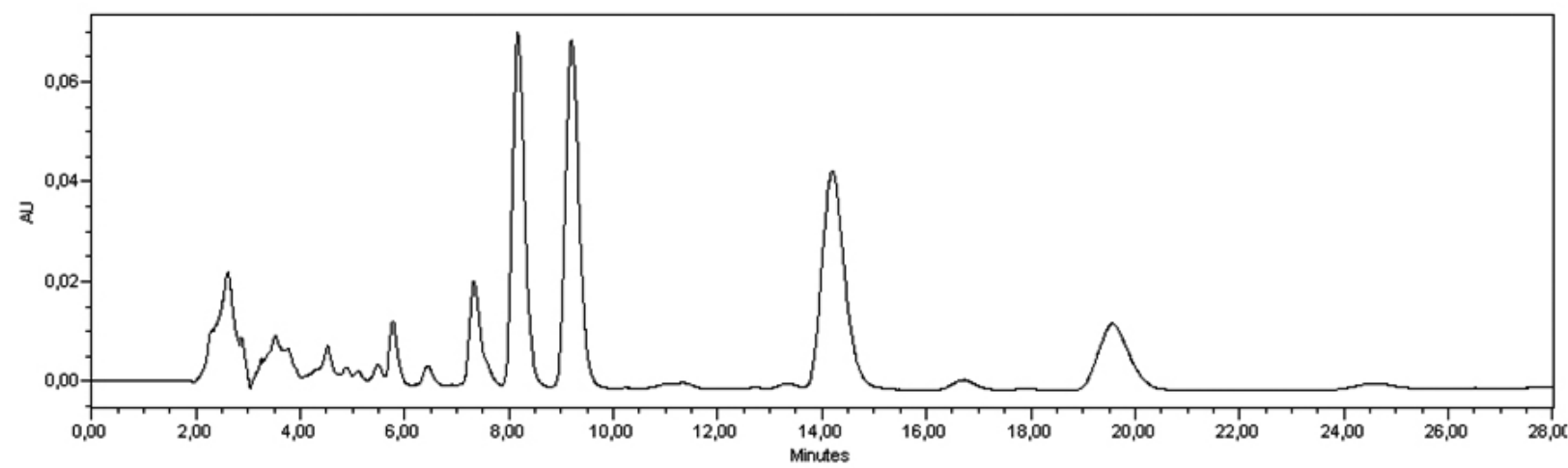

Figure 1: Chromatogram of Lonquén soil amended with a standard mixture of $10 \mathrm{mg} \mathrm{L}^{-1}$ of each anti-inflammatory. $\mathrm{t}_{\mathrm{R}:}$ Ketoprofen 8.1 min, Naproxen 9.2 min, Diclofenac $14.2 \mathrm{~min}$ and Ibuprofen $19.6 \mathrm{~min}$.

Table 3. Parameters of Freundlich, Langmuir and Kd for adsorption of NSAIDs in Linderos soil.

\begin{tabular}{|c|c|c|c|c|c|}
\hline \multicolumn{2}{|c|}{ Parameters } & Ketoprofen & Naproxen & Diclofenac & Ibuprofen \\
\hline \multirow{4}{*}{ Freundlich } & $\mathbf{K} \mathbf{d}_{\left(\mathrm{Lg}^{-1}\right)}$ & 0.0419 & 0.0398 & 0.0422 & 0.0200 \\
\hline & $\left.\mathbf{K} \mathbf{f}_{(\mathrm{mg}}{ }^{\mathrm{n}-1} \mathrm{~L}^{\mathrm{n}} \mathrm{g}^{-1}\right)$ & 0.0579 & 0.0337 & 0.0806 & 0.0154 \\
\hline & Nf & 0.7959 & 0.9945 & 0.6108 & 1.0371 \\
\hline & $\mathbf{R}^{2}$ & 0.8310 & 0.7867 & 0.9303 & 0.8289 \\
\hline \multirow{3}{*}{ Langmuir } & $\mathbf{V m} \mathbf{m g ~ g}^{-1}$ & 2.9674 & -0.0008 & 2.0947 & -2.6344 \\
\hline & $\mathbf{K}_{\left(\mathrm{Lg}^{-1}\right)}^{-1}$ & 0.0128 & -0.0035 & 0.0183 & -0.0049 \\
\hline & $\mathbf{R}^{2}$ & 0.0791 & 0.0062 & 0.4499 & 0.0516 \\
\hline
\end{tabular}

Mobility of NSAIDs in Linderos and Lonquén soils

Low mobility in the soil profile was observed for NSAID according to the results obtained, for both soils enriched with NSAIDs not treated with biosolid (Table 4). A decrease in the amount of each anti-inflammatory detected in the soil column profile was found. pKa of the NSAIDs are between 4.15 and $4.91^{6}$ thus these will be found in neutral form in soils under study ${ }^{22}$ and also have low polarity (log Kow between 3.12 and 4.51 ), presenting a greater interaction with the soil and therefore a low mobility in the soil profile.

Table 4. NSAIDs content in Lonquén and Linderos soils, Lonquén and Linderos soils amended with biosolids and Lonquén and Linderos sterilized soils.

\begin{tabular}{|c|c|c|c|c|c|c|c|c|}
\hline \multicolumn{5}{|c|}{ LONQUÉN } & \multicolumn{4}{|c|}{ LINDEROS } \\
\hline \multicolumn{9}{|c|}{ Soil $\left(\mathbf{m g ~ K g} \mathbf{g}^{-1}\right)$} \\
\hline Section & Ket & Nap & Dic & Ibu & Ket & Nap & Dic & Ibu \\
\hline 1 & 0.311 & 0.243 & nd & 0.175 & 0.174 & 0.461 & 0.071 & 0.500 \\
\hline 2 & 0.064 & 0.073 & nd & 0.030 & 0.036 & 0.107 & nd & 0.384 \\
\hline 3 & 0.020 & nd & nd & nd & 0.026 & nd & nd & 0.082 \\
\hline 4 & nd & nd & nd & nd & 0.019 & nd & nd & 0.015 \\
\hline \multicolumn{9}{|c|}{ Soil + biosolid (mg Kg-1) } \\
\hline Section & Ket & Nap & Dic & Ibu & Ket & Nap & Dic & Ibu \\
\hline 1 & 0.090 & 0.191 & nd & 0.751 & 0.310 & 0.458 & 0.228 & 0.665 \\
\hline 2 & 0.013 & 0.081 & nd & 0.179 & 0.099 & 0.080 & nd & 0.201 \\
\hline 3 & nd & nd & nd & nd & 0.027 & nd & nd & 0.100 \\
\hline 4 & nd & nd & nd & nd & nd & nd & nd & nd \\
\hline \multicolumn{9}{|c|}{ Sterilized soil (mg Kg $\left.{ }^{-1}\right)$} \\
\hline Section & Ket & Nap & Dic & Ibu & Ket & Nap & Dic & Ibu \\
\hline 1 & 0.064 & nd & 0.027 & 0.067 & 0.189 & nd & 0.082 & 0.094 \\
\hline 2 & 0.052 & nd & 0.005 & 0.057 & 0.100 & nd & nd & 0.067 \\
\hline 3 & 0.033 & nd & nd & 0.032 & 0.080 & nd & nd & 0.049 \\
\hline 4 & nd & nd & nd & 0.022 & 0.059 & nd & nd & 0.017 \\
\hline
\end{tabular}


In Lonquén soil, ketoprofen showed the greatest mobility, being detected as far as the third section, while naproxen and ibuprofen were only found until the second section, but in lower amounts than ketoprofen. No diclofenac was detected in the column; that may be deduced that they showed greate degradation, due to presence of microorganisms or to photodegradation/ oxidation process. Lin and Gan found that biodegradation of NDAIS in soils with similar characteristics (clay sandy loamy) follow the order: diclofenac $>$ ibuprofen $>$ naproxen. ${ }^{22}$ While in studies by Lin and Reinhard in water ${ }^{23}$, it was demonstrated that naproxen, ketoprofen, and ibuprofen exhibited photodegradation, being ketoprofen the fastest to break down.

A different behavior was observed in Linderos soil, where all antiinflammatories were detected; Ketoprofen and ibuprofen up to the four section, naproxen was up to the second section, while Diclofenac showed no mobility, and it was only detected in the first section, at very low concentration, probably due also to rapid photodegradation and/or biodegradation mainly governed by the soil matrix components. ${ }^{22}$

Linderos soil showing greater mobility of NSAIDs than Lonquén soil, and also higher total concentration through the column, could be attributed to the lower content of organic matter in the Linderos soil which would diminish biodegradation and fixation of analytes in the profile of the soil columns.

Mobility of NSAIDs in Linderos and Lonquén soils amended with biosolid

The mobility of NSAIDs in the leaching columns of the Lonquén soil enriched with biosolid (200 $\left.\mathrm{Mg} \mathrm{ha}^{-1}\right)$ is shown also in Table 4. Biosolid addition showed, for ketoprofen and naproxen, a respective decrease of $71 \%$ and $21 \%$ in the first column section, while in the second section the decrease reached $80 \%$ for ketoprofen. Thus, it may be inferred that addition of biosolid to the Lonquén soil decreased the amount of ketoprofen and naproxen found in each column section as a result of the content of organic matter in the biosolid, which could increase microbial activity of the system that contributes to breakdown of these products. In the case of ibuprofen, the content increased in sections 1 and 2 with biosolid addition, which may be mainly attributed to the structure of this analyte, since it presents lower steric hindrance than the other compounds under study, and this would allow its fixing to the organic matter of the added biosolid.

In Linderos soil treated with biosolid (Table 4), the amount of naproxen is similar to that extracted from soil without biosolid addition; moreover, the addition of biosolid increased the content of ketoprofen and diclofenac. As regards ibuprofen, a slight increase was found in its retention in the first column section.

Mobility of NSAIDs in sterilized Linderos and Lonquén soils

Razavi et al., found that sterilization of soils increase optical density, $\mathrm{pH}$, electrical conductivity, ion exchange capacity of the soil and increase the soluble organic acids ${ }^{24}$, those modifications would impact the mobility of the analytes in study. In general, in Lonquén soil the amounts of NSAIDs detected in first section decreased considerably after soil sterilization (Table 4). Ketoprofen concentration decreased in $79 \%$ in section 1, ibuprofen decreased in $62 \%$ and naproxen decreased in $100 \%$. It is probable that, as a result of the high pressures and temperatures necessary for to sterilization of soil, its composition may be modified, and fewer sites are available to retain these compounds.

In sterilized Linderos soil (Table 4) ibuprofen concentration decreased in $81 \%$ and naproxen decreased in $100 \%$ in section 1 . The amount of ketoprofen along the column increased compared with the same system withou sterilization, possibly due to decrease in microbial activity in the lower soil profile and a consequently lower degradation, thus increasing their presence. In this case, mobility would increase as a result of an increase in the amount retained in the column.

\section{CONCLUSIONS}

The method implemented for ultrasound-assisted solvent extraction in soil enriched with NSAIDs showed good recovery, with values ranging from $68 \%$ to $99 \%$, which indicates that the procedure in use permits adequate determination, and prove to be a significant tool for further studies.

In the studies of mobility of NSAIDs in the leaching columns without biosolid amendment, it was possible to determine ketoprofen, naproxen and ibuprofen, which were found in the first column sections. Instead, diclofenac determination was not possible, which may be attributed to ready biodegradation. Biosolid application to soils caused a decrease in NSAIDs content in lonquén soil, possibly due to an increase in their breakdown as a result of an increase in microbial activity. On the other hand, there was an increase in the content of ibuprofen in the first fraction of the Lonquén soil, which indicates that biosolid application would favor its retention in this soil, with a consequent decrease in leaching of this compound to groundwater. In Linderos soils addition of biosolids to Linderos soil generally increased antiinflammatory content. Anti-inflammatory mobility in the profile of soil depends on the specific characteristics of each system soil-biosolid.

In general, the studies with sterilized soils showed a decrease in the amount of NSAIDs along soil column, indicating that the sterilization method used affects the soil structure, leaving fewer sites available for the retention of anti-inflammatories drugs.

\section{ACKNOWLEDGEMENTS}

The authors thank FONDECYT Project 11090287 for financial support

\section{REFERENCES}

1.- R. Hudec, L. Bozeková, J. Tisonová, J Clin Pharm Ther, 37, 78-80, (2012)

2.- B. Crouse, A. Ghoshdastidar, A. Tong, Environ Res, 112, 92-99, (2012)

3.- G. Bori, B. Hernández, M. Gobbo, Á. Lanas, M. Salazar, L. Terán, J. Díaz, R. Espinosa, F. Galván, L. García, J. Álvarado-Garcia, H. Ávila, L. Carmona, J. Rivera, L. Ventura, Reumatol Clin. 5, 3, (2009)

4.- O. Jones, J. Lester, N. Voulvoulis, Trends Biotechnol. 23, 163, (2005)

5.- A. Azzouz, E. Ballesteros, Sci Tot Environ 419, 208-215, (2012)

6.- M. Gross, M. Petrović, D. Barceló, Talanta. 70,678, (2006)

7.- M. Gómez, M. Petrovic, R. Amadeo, D. Barceló, J Chromatogr. 1114, 224 (2006)

8.- M. Díaz-Cruz, D. Barcelo, Trac Trends in Anal Chem. 24, 645, (2005)

9.- M. Stumpf, T. Ternes, R. Wilken, S. Rodrigues, W. Baumann, Sci. Total Environ. 225, 135, (1999)

10.- E. Golet, A. Alder, A. Hartmann, T. Ternes, W. Gigger, Anal. Chem. 73 3632, (2001)

11.- T. Ternes, P. Kreckel, J. Mueller, Sci. Total Environ. 225, 91, (1999)

12.- V. Burkinaa, V. Zlabeka, G. Zamaratskaiaa, Environ Toxicol Phar, 40, 430-444, (2015)

13.- D. Carter, D. Yellowlees, M. Tibbett, Pedobiologia, 51, 295, (2007)

14.- A. Sadzawka, M. Carrasco, R. Grez, M. Mora, P. Flores, A.Neaman Métodos de análisis recomendados para suelos de Chile. Instituto de Investigaciones Agropecuarias, Serie Actas INIA-N³4, Santiago (2006)

15.- L. Kunde, J. Gan, Chemosphere. 83, 240, (2011)

16.- C. Leiva, I. Ahumada, B. Sepúlveda, P. Richter, Chemosphere. 79, 273, (2010)

17.- T. Ternes, M. Bonerz, N. Herrmann, D. Löffler, E. Keller, B. Bagó, A Alder, J Chromatogr A. 1067, 213, (2005)

18.- J. Santos, I. Aparicio, E. Alonso, M. Callejón, Anal Chim Acta. 550, 116 , (2005)

19.- L. Ascar, I. Ahumada, A. López, F. Quintanilla, K. Leiva, J Brazil Chem Soc. 24, 1160, (2013)

20.- C. Giles, T. MacEwan, S. Nakhwa, D. Smith, J. Chem. Soc. 111, 3973 (1960)

21.- J. Xu, L. Wu, A. Chang, Chemosphere. 77, 1299, (2009)

22.- K. Lin, J. Gan, Chemosphere. 83, 240, (2011)

23.- A. Lin, M. Reinhard, Environ Toxicol Chem. 24, 1303, (2005)

24.- S. Razavi darbar, A. Lakzian, J Environ Sci. 5, 87, (2007) 